\title{
The Shape of Silicate Features in Semiregular and Mira Variables
}

\author{
JOSEF HRON, BERNHARD ARINGER, \\ and FRANZ KERSCHBAUM
}

Institut für Astronomie der Universität Wien, Vienna, Austria

We have analyzed the mid-IR silicate emission features from O-rich semiregular (SR) and Mira variables by fitting blackbodies to the underlying photospheric and dust continua and computing ratios of the excess flux in selected wavelength bands. These ratios were then related to the photospheric temperatures and the optical depths of the dust shells. The ratio of the strengths of the $10 \mu \mathrm{m}$ and $18 \mu \mathrm{m}$ features decreases from the SRs to the Miras. Narrow $10 \mu \mathrm{m}$ features are found for the 'hottest' stars (mostly SR) and for cool stars with thick envelopes (mostly Miras). Thus optical depth effects can almost certainly be ruled out as the main factor influencing the feature shapes. We discuss possible explanations in terms of current ideas on the formation of dust in oxygen-rich stars.

This work is supported by the Fonds zur Förderung der wissenschaftlichen Forschung under project number S7308-AST. 